Universität Bielefeld Faculty of Business Administration and Economics

Working Papers in Economics and Management

No. 04-2018

February 2018

\title{
Delaying Product Introduction: A Dynamic Analysis with Endogenous Time Horizon
}

Serhat Gezer 


\title{
Delaying Product Introduction:
}

\section{A Dynamic Analysis with Endogenous Time Horizon}

Serhat Gezer*

February 7, 2018

\begin{abstract}
We consider a capital accumulating incumbent firm which produces an established product and has the option to introduce an improved substitute product to the market by incurring adoption costs. We find that depending on the initial capacities on the established market and the value of adoption costs, three scenarios are possible, namely introducing immediately, later or abstaining from product introduction. In case of delay of product introduction, the incumbent reduces capacities for the established product before the new product is introduced. We encounter Skiba points where the incumbent is indifferent between two of the three scenarios and use a bifurcation analysis in order to characterize the transition towards different steady states.
\end{abstract}

JEL classification: D42; L12; O31

Keywords: Product innovation, Capacity dynamics, Multi-product firm, Free endtime problem, Delayed product introduction

\footnotetext{
${ }^{*}$ Department of Business Administration and Economics and Center for Mathematical Economics, Bielefeld University, Universitätsstraße 25, 33615 Bielefeld, Germany, E-mail address: sgezer@uni-bielefeld.de
} 


\section{Introduction}

For many firms, especially those operating in the high-tech sector, whenever a new technology is available, they have to decide whether to adjust the product range by incorporating the new technology and if yes, when to do so.

Wang and Hui (2012) provide examples of firms hesitating to incorporate new available technologies and choosing to stay with the old technology for a while. Examples include the technology of DVD that has been developed much earlier than vendors started promoting DVDs. Another example is the MP3 standard.

In an empirical investigation, Chandy and Tellis (2000) have found that a large fraction of product innovations has been achieved by incumbents. Indeed, we face such a situation described above often in real-world markets and in many industries, submarkets evolve and coexist with the established product. An example is the TV Industry where CRT televisions and flatscreens were sold simultaneously for a long time (cf. Dawid et al. (2015)).

We consider an incumbent firm which has the option to introduce a horizontally and vertically differentiated substitute product which has a higher quality than the established one. For realizing this option, it incurs one-time adoption costs. Thus, the firm has to determine if the product introduction is profitable and if yes, when the optimal time of product introduction is. After introduction, we assume that the firm sells both products.

The firm faces the following trade-off: At the one hand, by launching the new product it cannibalizes demand for the established product and at the other hand, it benefits from the new product with higher quality by exploiting higher willingness to pay of the consumers. We find that the cannibalization effect alone cannot cause a delay. Delay is optimal if and only if there are adoption costs as well e.g. coming from adjustment costs of the plant, advertisement activities or fees paid to developers for using their technologies.

In particular, we find that if the firm is strong at the established market, i.e. its capacities are at a high level, then the firm decides to wait and hence to introduce the improved product later. By delaying, the firm benefits from discounting adop- 
tion costs while it decreases the capacity of the established product before the new product is introduced. This reduction of capacity increases the marginal values of the capacities of the established and the new product at the time of product introduction. Amongst others, this enables the incumbent to build-up capacities for the new product faster when it is introduced, compared to immediate introduction.

There is a large literature on capital accumulating firms which has been extended by Dawid et al. (2015) who analyzed the optimal R\&D effort for product innovation and capital accumulation of established and new products, where the breakthrough probability of developing a new product depends on both, a knowledge stock and current $R \& D$ efforts via a hazard rate. Hence, in that paper innovation time is stochastic and it is assumed that the new product is introduced immediately once it is available. We focus on the optimal timing of product introduction and optimal investment in capacities and differ from Dawid et al. (2015) in not considering R\&D efforts to develop a new product and not linking successful development to market introduction but considering the time of market introduction as a choice variable. The classical literature on optimal timing of technology adoption (see. e.g. Kamien and Schwartz (1972) for a single firm and Reinganum (1981) and Fudenberg and Tirole (1985) for a duopoly) assumes that quality increases due to technological progress and the only decision variable is the time of technology adoption. Farzin et al. (1998) and Doraszelski (2004) extend this stream of literature by considering the quality improvement as a stochastic process. In contrast, in our model, the quality of the new product is fixed and the firm cannot gain additional quality by delaying. Thus, our analysis focuses on the dependence on initial characteristics whose importance has been addressed a lot, e.g. in Hinloopen et al. (2013) where initial marginal costs determine if a technology is developed further or not. Real options models (see e.g. Dixit and Pindyck (1994)) have focussed on optimal timing in continuous time where demand is stochastic e.g. evolving according to a Brownian motion. A simultaneous analysis of optimal timing and optimal investment in capacities in the real options literature has been provided by Huisman and Kort (2015) where the price of the good is stochastic. We differ from that stream of literature by considering a deterministic environment and 
continuous adjustments of capacities.

The problem of an incumbent delaying product introduction has been addressed in Wang and Hui (2012). They apply a discrete three-period time framework where they do not take into account capacity adjustments. In contrast to Wang and Hui (2012), in our model, delaying cannot be optimal if there are no adoption costs.

From a technical perspective, we employ Pontryagin's Maximum Principle for free end time (see. e.g. Grass et al. (2008)) to obtain analytical results concerning the optimal investments and the optimal time of market introduction.

Moreover, in this optimal control problem, due to the non-concave structure of the value function, the Arrow-Mangasarian sufficiency conditions are not met which for certain states lead to the presence of multiple optimal investment paths. In particular, we characterize situations in which the firm is indifferent between approaching different steady states (see Skiba (1978)). In such models, qualitative properties of solutions depend very much on parameters (cf. Hinloopen et al. (2013)). Therefore, we use a bifurcation analysis to assess industry dynamics for different values of adoption costs where we encounter a deformed pitchfork bifurcation.

The analysis in this paper is carried out for a monopoly setting. Even though the real-world examples we have raised stem from competitive environments, we believe that it is important to consider the monopoly as it is interesting in its own right. Indeed, timing of product introduction is not only influenced by competing firms but from competing substitute products as well even if there is only a single firm. As the established and new product are substitutes, there is 'internal' competition between those two products. In order to disentangle rivalry between products and between firms, it is reasonable to analyze the monopoly case before proceeding to the competition case.

The paper is organized as follows. We introduce the model in Sect. 2. Sect. 3 is devoted to the technical analysis. In Sect. 4, we provide an economic interpretation, conduct a bifurcation analysis and present optimal timing curves. Sect. 5 analyzes welfare effects of delaying product introduction. Model assumptions are discussed in Sect. 6 and Sect. 7 concludes. 


\section{Model}

We consider an incumbent firm which has initial capacity $K_{1}^{i n i}$ to produce an established product. A new substitute product with higher quality has been developed and is ready for market introduction. Product introduction comes with lump-sum adoption costs $F$. An important assumption is that the incumbent cannot invest in capacities of the new product before introducing it, i.e. there are no capacities at the time of introduction for the new product.

We follow the literature on optimal capital accumulation by relying on a standard linear model (see e.g. Dockner et al. (2000)). Thus, the firm faces a linear inverse demand function which is given by

$$
p_{1}(t)=1-K_{1}(t)
$$

After product introduction, the inverse linear demand system ${ }^{1}$ is given by

$$
p_{1}(t)=1-K_{1}(t)-\eta K_{2}(t)
$$

and

$$
p_{2}(t)=1+\theta-\eta K_{1}(t)-K_{2}(t)
$$

where $\eta$ with $0<\eta<1$ measures the degree of horizontal and $\theta>0$ the degree of vertical differentiation of the substitutes.

The firm wants to determine the optimal time of product introduction $T$ and the optimal investment strategies before and after product introduction. There is no inventory, i.e. capacities equal sales ${ }^{2}$. The capacity dynamics are

$$
\begin{gathered}
\dot{K}_{i}(t)=I_{i}(t)-\delta K_{i}(t), \quad i=1,2, \\
K_{1}(0)=K_{1}^{i n i}, \quad K_{2}(t)=K_{2}^{i n i}=0 \forall t \leq T,
\end{gathered}
$$

\footnotetext{
${ }^{1}$ This demand system is motivated by the fact that the two products are substitutes and competing with each other. According to the seminal result of Kreps and Scheinkman (1983), setting prices optimally subject to ex-ante capacity commitments reduces to a Cournot setting which we adopt here.

${ }^{2}$ This assumption has been used in large parts of the literature on dynamic capacity investment, see e.g. Goyal and Netessine (2007). See Section 6 for a discussion of this assumption.
} 
where $\delta>0$ measures the depreciation rate. As has been done in Dawid et al. (2015), we allow the firm to intentionally scrap capacities, i.e. $I_{i} \in \mathbb{R}$ while capacities have to remain non-negative:

$$
K_{i}(t) \geq 0 \quad \forall t \geq 0, i=1,2
$$

Adjusting capacities is costly, in particular it comes with quadratic costs

$$
C\left(I_{i}(t)\right)=\frac{\gamma}{2} I_{i}^{2}(t), \quad i=1,2
$$

Normalizing production costs to zero, the objective function of the firm is given by the following expression:

$$
\begin{aligned}
\max _{T, I_{1}(t), I_{2}(t)} J & =\int_{0}^{T} e^{-r t}\left(p_{1}(t) K_{1}(t)-C\left(I_{1}\right)\right) d t \\
& +\int_{T}^{\infty} e^{-r t}\left(p_{1}(t) K_{1}(t)+p_{2}(t) K_{2}(t)-C\left(I_{1}\right)-C\left(I_{2}\right)\right) d t-e^{-r T} F
\end{aligned}
$$

We refer to this problem as $\mathcal{P}\left(K_{1}^{i n i}\right)$.

\section{Analysis}

In case that the firm wants to introduce the improved product at some finite time $T$, there will be a structural change of the model. Therefore, we denote by mode 1 $\left(m_{1}\right)$ the optimal control problem up to time $T$ and by mode $2\left(m_{2}\right)$ the problem after $T$. Denote by $V^{m_{1}}\left(K_{1}\right)$ and $V^{m_{2}}\left(K_{1}, K_{2}\right)$ the corresponding value functions of the infinite horizon control problems where the mode is fixed and hence does not change $^{3}$. The optimal control problem at hand where the mode $m$ might change is denoted by $V\left(K_{1}, K_{2}, t, m\right)$ and we refer to this problem as the optimal control problem with introduction option.

The subproblem in $m_{2}$ is linear-quadratic with infinite time horizon which can be solved easily, as has been done in Dawid et al. (2015). The optimal strategy and the value function are stationary for this problem, i.e. $V\left(K_{1}, K_{2}, t, m_{2}\right)=V^{m_{2}}\left(K_{1}, K_{2}\right)$. There is a unique globally asymptotically stable steady under the optimal strategy

\footnotetext{
${ }^{3}$ We suppress the argument $t$ wherever it is possible and does not cause confusion.
} 
and the value function is given by ${ }^{4}$

$$
V^{m_{2}}\left(K_{1}, K_{2}\right)=a+b K_{1}+c K_{1}^{2}+d K_{2}+e K_{2}^{2}+f K_{1} K_{2}
$$

The typical shape of the value function of $m_{2}$ is depicted in Figure 1.

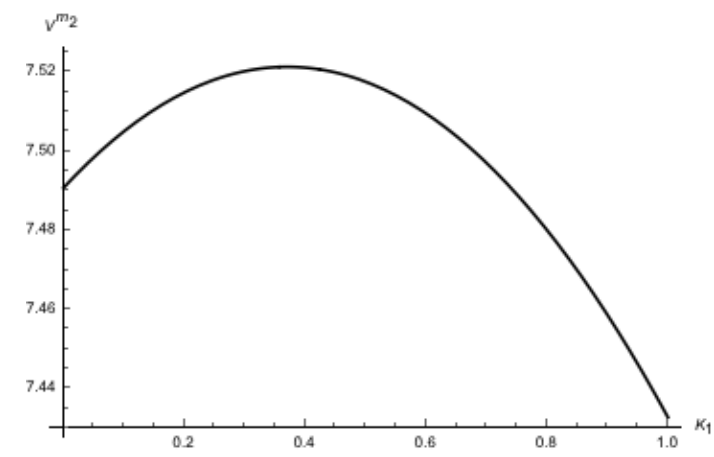

Figure 1: Value function of $m_{2}$ at $T$, i.e. for $K_{2}=0$. Parameters: $r=0.04, \delta=$ $0.1, \eta=0.9, \theta=0.1, \gamma=0.15$.

By regarding the value function of the subproblem as the salvage value of the optimal control problem with introduction option, we can rewrite (8) by

$$
\max _{T, I_{1}(t)} J=\int_{0}^{T} e^{-r t}\left(p_{1}(t) K_{1}(t)-C\left(I_{1}(t)\right)\right) d t+e^{-r T} S\left(K_{1}(T)\right),
$$

where $S\left(K_{1}(T)\right)=V^{m_{2}}\left(K_{1}(T), 0\right)-F .{ }^{5}$ This problem can be solved analytically by Pontryagin's Maximum Principle for variable terminal time. The Hamiltonian is

$$
H\left(K_{1}, I_{1}, \lambda, t\right)=\left(1-K_{1}\right) K_{1}-\frac{\gamma}{2} I_{1}^{2}+\lambda\left(I_{1}-\delta K_{1}\right),
$$

where $\lambda$ is the co-state variable and the optimal investment is given by

$$
I_{1}=\frac{\lambda}{\gamma}
$$

The co-state equation reads

$$
\dot{\lambda}=(r+\delta) \lambda-\left(1-2 K_{1}\right),
$$

and the transversality condition is given by ${ }^{6}$

$$
\lambda(T)=S_{K_{1}}=V_{K_{1}}^{m_{2}}\left(K_{1}, 0\right) .
$$

\footnotetext{
${ }^{4}$ Equations for coefficients are given in Dawid et al. (2015).

${ }^{5} K_{2}(T)=0$ since there are no capacities for the new product at $T$, yet.

${ }^{6}$ The canonical system, isoclines, the steady state for staying in $m_{1}$ and its stability properties are given in Appendix A.1.
} 
For nonzero finite $T^{*}$, let $\left(K_{1}^{*}(\cdot), I_{1}^{*}(\cdot)\right)$ be an optimal solution to (10) on the optimal time interval $\left[0, T^{*}\right]$. Pontryagin's Maximum Principle for variable end time implies an additional constraint for the terminal time, which is given by

$$
H\left(K_{1}^{*}\left(T^{*}\right), I_{1}^{*}\left(T^{*}\right), \lambda\left(T^{*}\right), T^{*}\right)=r S\left(K_{1}^{*}\left(T^{*}\right)\right)-S_{T}\left(K_{1}^{*}\left(T^{*}\right)\right) .
$$

Note that the salvage value does not depend explicitly on $T^{*}$ and hence,

$$
S_{T}\left(K_{1}^{*}\left(T^{*}\right)\right)=0 .
$$

So, equation (15) requires that at the optimal time $T^{*}$, the instantaneous revenue from staying in $m_{1}$ plus the assessment of the change of the state variable on the one hand (which is given by the current-value Hamiltonian, abbr. by $H$ ) and the interest on the salvage value (abbr. by $r S$ ) on the other hand are equal. This is quite intuitive since otherwise it would be optimal to stay longer in $m_{1}$ if $H$ is higher than $r S$ or to have introduced earlier if $r S$ is higher than $H$.

In Lemma 2 in Appendix A.2, we state that there are two solutions for equation (15). By that lemma and Proposition 1 below, we show that for $F=0$, both solutions coincide and $H \leq r S$ for all values of established capacity, i.e. immediate introduction is optimal and hence $T^{*}=0$. For $F>0$, there are two distinct points satisfying the terminal condition. In the corresponding interval, where the boundaries are given by the two points satisfying (15), there is $H \geq r S$ (cf. Figure 12 in Appendix A.2), i.e. for initial capacities in the interval, it is optimal to reduce capacities down to the lower bound and to introduce the new product, we say to jump to $m_{2}$. We denote the two solutions of (15) by $K_{1}^{l b}$ and $K_{1}^{u b}$, respectively for lower and upper bound of the interval with $K_{1}^{l b} \leq K_{1}^{u b}$. As mentioned above, for $F=0$, both solutions coincide ${ }^{7}$, i.e. $K_{1}^{l b}=K_{1}^{u b}$ (see Appendix A.2), which we denote by $K_{1}^{F=0}$.

So, for higher capacities than $K_{1}^{u b}$, the unique solution is to introduce the new product immediately again. In particular at $K_{1}^{u b}$, the firm is indifferent between

\footnotetext{
${ }^{7}$ Technically, in case of no adoption costs, $H$ and $r S$ are tangential at $K_{1}^{F=0}$ :

$$
\frac{\partial}{\partial K_{1}} H\left(K_{1}^{F=0}, I_{1}^{*}\left(T^{*}\right), \lambda\left(T^{*}\right), T^{*}\right)=\frac{\partial}{\partial K_{1}} r V^{m_{2}}\left(K_{1}^{F=0}, 0\right) .
$$


both options. However, higher capacities than $K_{1}^{u b}$ will not be analyzed further as there the firm switches immediately to $m_{2}$ which has been analyzed in Dawid et al. (2015).

As the optimal introduction time depends on the size of capacity, we consider it as a correspondence depending on $K_{1}^{i n i}$ and denote it by $T^{*}\left(K_{1}^{i n i}\right)^{8}$. It is a correspondence since there are situations with multiple optimal values as we will discuss in the following. We start by characterizing finite solutions.

Proposition 1. If $T^{*}\left(K_{1}\right)$ is finite for all $K_{1}$, then for all $K_{1} \leq K_{1}^{l b}$, it is optimal to innovate immediately. For all $K_{1}^{l b}<K_{1} \leq K_{1}^{u b}$, it is optimal to reduce capacities and to innovate when the capacity reaches $K_{1}^{l b}$, i.e. $T^{*}\left(K_{1}\right)>0$.

Proof. See Appendix A.3

Proposition 1 states that immediate introduction is optimal if capacity for the established product is lower than a certain threshold (given by $K_{1}^{l b}$ ) whereas for capacities above, it is optimal to wait and to decrease capacities on the established market before product introduction. Note, that there are infinite solutions where it is not optimal to innovate immediately even though $K_{1}^{i n i} \leq K_{1}^{l b}$ as we will discuss at the end of this section.

In the next lemma we focus on the dependence of $K_{1}^{l b}$ on $F$ and find that $K_{1}^{l b}$ is decreasing in $F$, i.e. as adoption costs increase, it takes longer to arrive at $K_{1}^{l b}$ for a fixed starting point $K_{1}^{i n i}>K_{1}^{l b}$.

Lemma 1. $K_{1}^{l b}$ is decreasing in $F .^{9}$

Proof. See Appendix A.4.

In Figure 2, we illustrate how the value function evolves as $F$ increases. For $K_{1}^{l b}<K_{1}<K_{1}^{u b}$, the value function of the problem with introduction option is higher than the value function of $m_{2}$. As $F$ increases and discounting adoption

\footnotetext{
${ }^{8} \mathrm{An}$ alternative would have been to define a function which gives the remaining time in $m_{1}$ not depending on the initial but current capacity (cf. Long et al. (2017)).

${ }^{9}$ Moreover, $K_{1}^{u b}$ is increasing in $F$. Thus, for increasing $F$, the interval $\left[K_{1}^{l b}, K_{1}^{u b}\right]$ expands around $K_{1}^{F=0}$.
} 


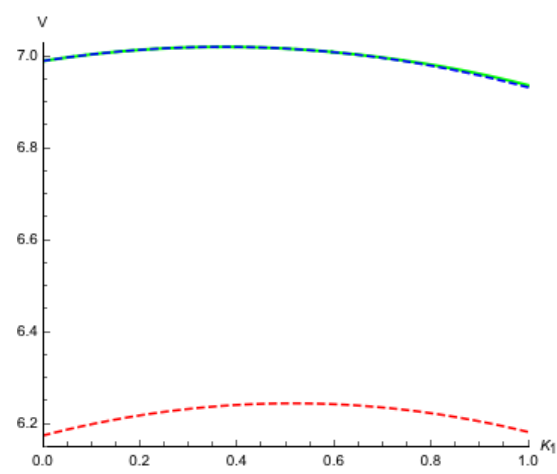

(a) $F=0.5$

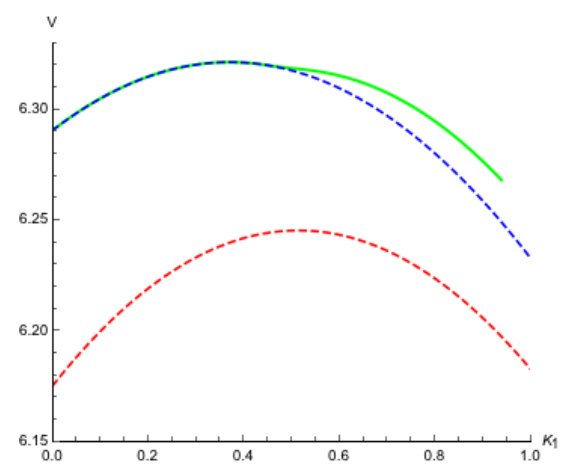

(c) $F=1.2$

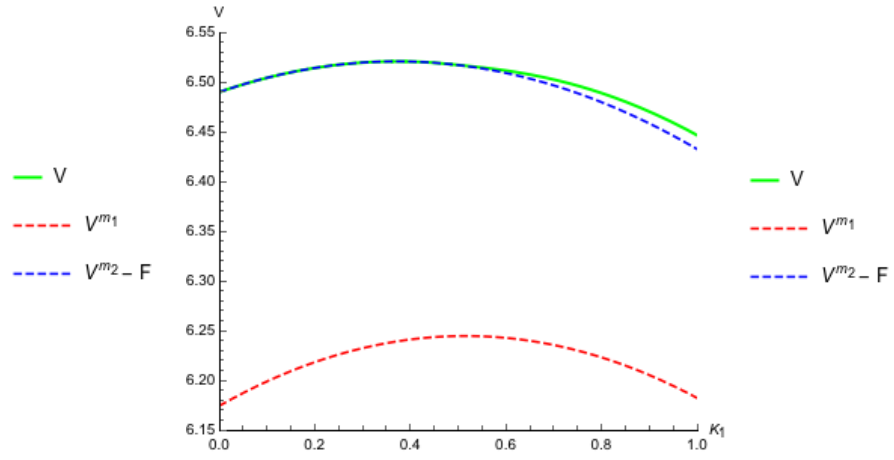

(b) $F=1$

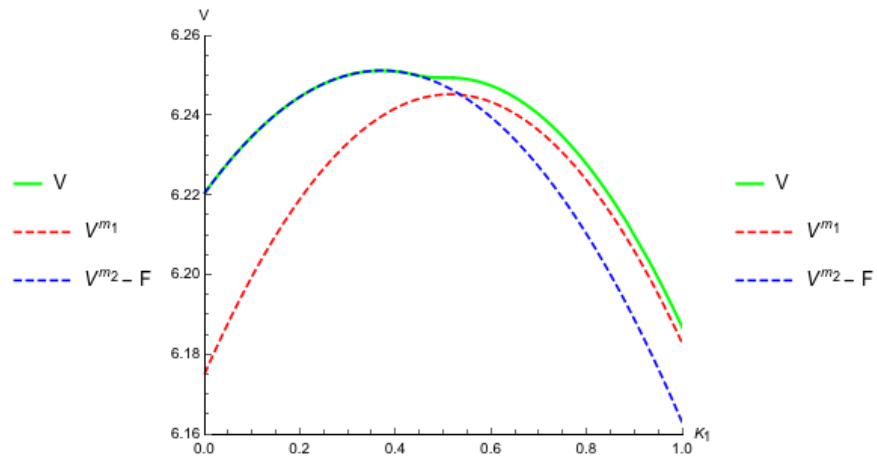

(d) $F=1.27$

Figure 2: Value functions for different values of $F$. Parameters: $r=0.04, \delta=$ $0.1, \eta=0.9, \theta=0.1, \gamma=0.15$.

costs become more important, the difference of the value function with introduction option and the scrap value function gets larger. Furthermore, as the products are vertically differentiated, the value of the problem of $m_{2}$ is higher than of $m_{1}$ for no adoption costs. Thus, the value of the problem with introduction option is higher than the value of the infinite problem of $m_{1}$. Obviously, for large enough $F$, the value function of the problem with introduction option will hit the value function of the infinite horizon problem of $m_{1}$ and infinite solutions will occur, i.e. product introduction will not be sufficiently attractive anymore. We show in Appendix A.5 in Lemma 3 that there exists a unique value of adoption costs $\tilde{F}$ where this happens for the first time (see Figure 3). Thus, $\tilde{F}$ is the lowest value of adoption costs for which it exists some initial value of capacity where the firm abstains from product introduction. This result leads to the following corollary.

Corollary 1. For $F<\tilde{F}, T^{*}\left(K_{1}\right)$ is finite for all initial capacities and Proposition 


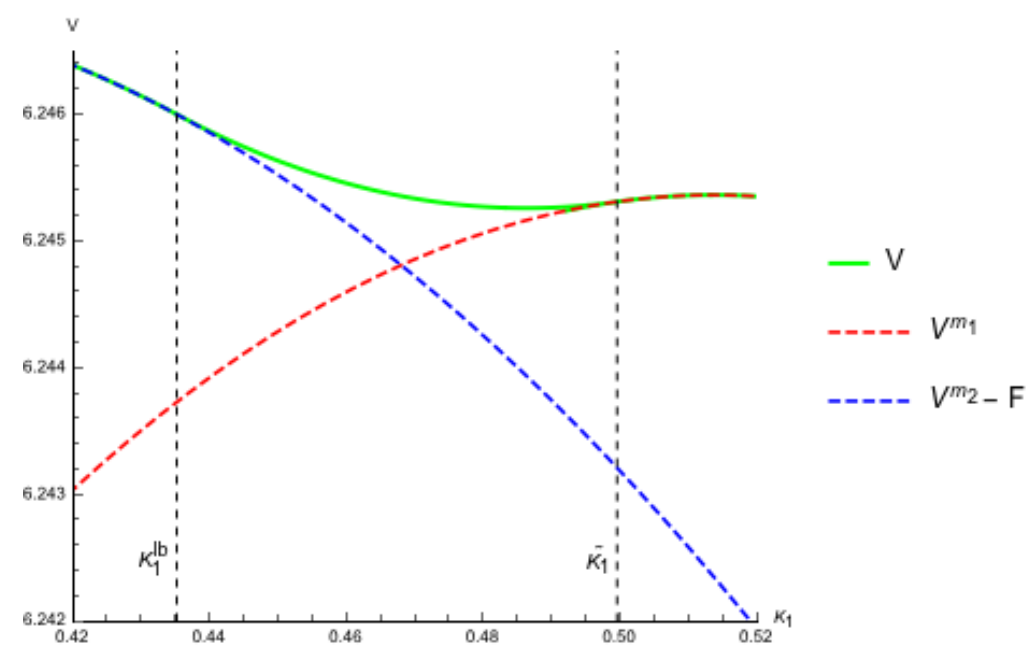

Figure 3: Value function for $F=\tilde{F}=1.27437$. Parameters: $r=0.04, \delta=0.1, \eta=$ $0.9, \theta=0.1, \gamma=0.15$.

\section{1 applies.}

Proof. Follows directly from Lemma 3 in Appendix A.5.

To sum up the results so far, for $F=0$, the firm wants to launch the new product immediately. For increasing $F$, there arises an interval given by $\left[K_{1}^{l b}, K_{1}^{u b}\right]$ wherein the higher $K_{1}^{i n i}$ the longer it takes to arrive at $K_{1}^{l b}$ where the firm wants to launch the new product, i.e. the stronger the firm on the established market, the more the firm delays. Moreover, due to Lemma 1, the higher the adoption costs, the lower is the switching capacity, i.e. the firm wants to reduce capacities more in advance before switching to $m_{2}$.

Denote by $\tilde{K}_{1}$ the lowest value of initial capacity where an infinite solution exists for $\mathcal{P}\left(\tilde{K}_{1}\right)$ :

$$
\tilde{K}_{1}=\min \left\{K_{1} \mid T^{*}\left(K_{1}\right)=\infty\right\} .
$$

Note that $\tilde{K}_{1}$ exists for $F \geq \tilde{F}$. The following proposition and corollary characterize the situation at $\tilde{F}$.

Proposition 2. At $F=\tilde{F}$,

$$
\tilde{K}_{1}=K_{1}^{s s, m_{1}} 10
$$

and the free end-time problem $\mathcal{P}\left(\tilde{K}_{1}\right)$ has a unique solution with $T^{*}=\infty$.

\footnotetext{
${ }^{10} K_{1}^{s s, m_{1}}$ is the unique steady state for staying infinitely in $m_{1}$ given in Appendix A.1.
} 
Proof. See Appendix A.7.

Corollary 2. At $\tilde{F}$, for $K_{1}<\tilde{K}_{1}$,

$$
T^{*}\left(K_{1}\right)<\infty
$$

and for $\tilde{K}_{1} \leq K_{1}<\bar{K}_{1}$,

$$
T^{*}\left(K_{1}\right)=\infty
$$

Proof. Due to the definition of $\tilde{K}_{1}$, for $K_{1}<\tilde{K}_{1}$ only finite solutions are optimal. According to the proof of Proposition 2 , for $\tilde{K}_{1} \leq K_{1}<K_{1}^{u b}$, only infinite solutions are optimal.

Proposition 2 and Corollary 2 state that at $\tilde{F}, K_{1}^{s s, m_{1}}$ is a threshold separating finite and infinite solutions. That is, for $K_{1} \geq K_{1}^{s s, m_{1}}$ the firm prefers not innovating and stays in $m_{1}$, whereas for $K<K_{1}^{s s, m_{1}}$ the firm decreases ${ }^{11}$ capacities to $K_{1}^{l b}$ and hence introduces the new product eventually.

For characterizing the evolution of $\tilde{K}_{1}$, we denote by $\bar{F}$ the value of adoption costs for which

$$
V^{m_{1}}\left(K^{l b}\right)=V^{m_{2}}\left(K^{l b}\right)-F\left(=S\left(K^{l b}\right)\right)
$$

holds, i.e. where the firm is indifferent between introducing immediately and delaying infinitely at $K_{1}^{l b}$.

Proposition 3. $\tilde{K}_{1}$ is decreasing in $F$ and for all $\tilde{F}<F<\bar{F}$, the free end-time problem $\mathcal{P}\left(\tilde{K}_{1}\right)$ has two different solutions with optimal terminal times $0<T^{f}<\infty$ and $T^{\infty}=\infty$, i.e. $\tilde{K}_{1}$ is a Skiba point where the firm is indifferent between introducing the product after some delay and not at all.

Proof. See Appendix A.8.

A consequence of Proposition 3 is that as $F$ increases, the range of capacities where the firm stays with only one product enlarges as $\tilde{K}_{1}$ decreases. Moreover, there is a finite and infinite solution at $\tilde{K}_{1}{ }^{12}$. As before, the timing for capacities

\footnotetext{
${ }^{11}$ In Appendix A.6 in Lemma 4, we show that at $\tilde{F}, K_{1}^{l b} \leq K^{s s, m_{1}}$ holds.

${ }^{12}$ There is no other value of capacity where both solutions are optimal.
} 
lower than $\tilde{K}_{1}$ is finite. So there exist three different ranges of capacities where optimal time of product introduction is either 0 , infinite or in-between. We refer to $[\tilde{F}, \bar{F})$ as the intermediate range of $F$ and for $F \in[\tilde{F}, \bar{F})$ we refer to $\left(K_{1}^{l b}, \tilde{K}_{1}\right)$ as the waiting region.

Denote by $\overline{\bar{F}}$ the value of adoption costs where thereafter finite solutions disappear for the first time ${ }^{13}$, i.e.

$$
T^{*}(0)=\infty
$$

Now, we show that at $\bar{F}$ the waiting region vanishes and only immediate or infinite solutions for $T$ remain.

Corollary 3. For $\bar{F} \leq F<\overline{\bar{F}}$, there exists a $\tilde{K}_{1}>0$ such that for all $K_{1}<\tilde{K}_{1}$ the firm introduces the new product immediately whereas for all $K_{1}>\tilde{K}_{1}$ the firm never introduces the new product. At $\tilde{K}_{1}$, the incumbent is indifferent, in particular the free end-time problem $\mathcal{P}\left(\tilde{K}_{1}\right)$ has two different solutions with $0=T^{f}<T^{\infty}=\infty$. Moreover, at $\bar{F}, \tilde{K}_{1}=K_{1}^{l b}$.

Proof. By definition of $\bar{F}$, the firm is indifferent between immediate and infinite product introduction. By Proposition $3, \tilde{K}_{1}$ is decreasing and hits $K_{1}^{l b}$ at $\bar{F}$ where solutions with $0<T<\infty$ vanish.

Thus, for all $F, \tilde{K}_{1}$ is separating finite and infinite solutions for $T$. Note that for $F<\bar{F}$, the value function of $m_{2}$ and the value function of the problem with introduction option paste smoothly at $K_{1}^{l b}$, i.e. ${ }^{14}$

$$
\frac{\partial V\left(K_{1}^{l b}, 0, m_{1}\right)}{\partial K_{1}}=\frac{\partial V^{m_{2}}\left(K_{1}^{l b}\right)}{\partial K_{1}} .
$$

Furthermore, at $\tilde{F}$, the value function of the problem with introduction option and the value function of $m_{1}$ paste smoothly at $\tilde{K}_{1}$ (see Figure 3 ) whereas for $F>\tilde{F}$ the value function has a kink at $\tilde{K}_{1}$ (cf. Figure 4 ).

\footnotetext{
${ }^{13} \mathrm{As} \tilde{K}_{1}$ is decreasing in $F$, at $\overline{\bar{F}}, K_{1}=0$ is the only remaining value for capacity such that the firm is indifferent between immediate and no product introduction.

${ }^{14}$ Note that the value function is time-invariant and hence the time argument can be omitted, i.e. $V\left(K_{1}^{l b}, K_{1}^{l b}, t, m\right)=V\left(K_{1}^{l b}, K_{1}^{l b}, m\right)$.
} 


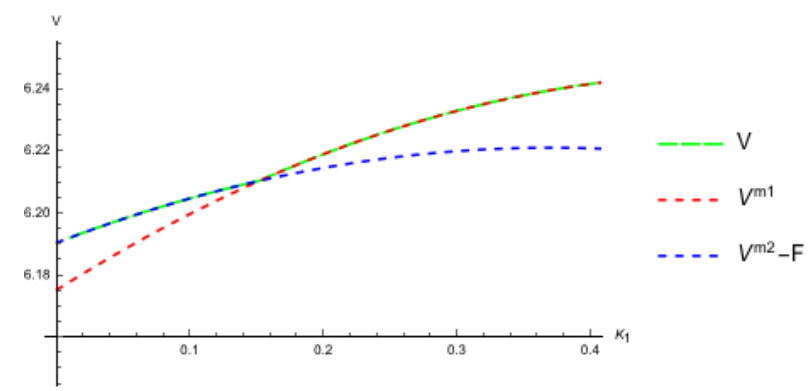

Figure 4: Value function for high $F$. Parameters: $r=0.04, \delta=0.1, \eta=0.9, \theta=$ $0.1, \gamma=0.15, F=1.3$.

\subsection{Summary of Results}

In total, as long as $F$ is intermediate (i.e. $\tilde{F} \leq F<\bar{F}$ ), we can split the state space in three parts:

i) 'Immediate introduction': $K_{1} \leq K_{1}^{l b}$ : Firm innovates immediately, $T^{*}=0$.

ii) 'Delayed product introduction': $K_{1}^{l b}<K_{1} \leq \tilde{K}_{1}$ : Firm delays introduction and introduces product later at $0<T^{*}<\infty$.

iii) 'No introduction': $K_{1} \geq \tilde{K}_{1}$ : Firm delays introduction infinitely, i.e. there is no product introduction.

For increasing $F$ the indifference point $\tilde{K}_{1}$ shifts to the left and eventually the waiting region vanishes where $\tilde{K}_{1}$ and $K_{1}^{l b}$ coincide and only two possibilities remain: Either the firm innovates immediately (for low capacities) or never (for high capacities). Hence, for $F \geq \bar{F}$, the value function is given by the upper curve of the value functions $V^{m_{1}}$ and $V^{m_{2}}$ (see Figure 4).

We call $F$ low if $0<F<\tilde{F}$, intermediate if $\tilde{F} \leq F<\bar{F}$, high if $\bar{F} \leq F \leq \overline{\bar{F}}$ and very high if $F>\overline{\bar{F}}$.

- If there are no adoption costs, only scenario i) is prevalent.

- For low adoption costs, scenarios i) and ii) are possible depending on the initial capacity level.

- If $F$ is intermediate, all three scenarios are possible. 
- For high adoption costs, only scenarios i) and iii) are possible.

- For very high adoption costs, only scenario iii) is prevalent.

\section{Dynamics}

In Section 4.1, we give an economic interpretation of the optimal capacity investments and the timing decision. A bifurcation analysis is presented in Section 4.2. Optimal timing curves and its dependence on parameters of horizontal and vertical differentiation are given in Section 4.3.

In order to derive dynamics, we consider the following default parameter setting taken from Dawid et al. (2015):

$$
r=0.04, \delta=0.1, \eta=0.9, \theta=0.1, \gamma=0.15
$$

\subsection{Economic Interpretation}

The intuition for the 'Immediate Introduction' and 'No Introduction' scenario is straight forward. The benefit from the new product is either so high that the firm does not want to wait or the benefit is too low such that the firm stays with the established product. Thus, we focus on the interpretation of the interesting case of delay. Note that for finite $T^{*}$, before $T^{*}$, the Hamiltonian $H$ is greater than the interest on the salvage value $r S$ and at $T^{*}$, they are equal ${ }^{15}$. In a sense the firm exploits profits in $m_{1}$ before moving to $m_{2}$. By choosing $T^{*}>0$, the Hamiltonian is affected ${ }^{16}$ via the co-state $\lambda(t)$. In economic terms, the following mechanisms can be identified.

First, the delay in time leads to stronger discounting of the scrap value $V^{m_{2}}-F$. The firm saves adoption costs as $F$ is paid as a lump-sum, but gets $V^{m_{2}}$ later as well. The latter is smoothed by the concave structure of the value function of $m_{2}$ as

\footnotetext{
${ }^{15}$ Note that this is not necessarily true for the infinite case since if $T^{*}$ is infinite, the transversality condition for the co-state variable and hence the Hamiltonian would be altered.

${ }^{16}$ Note that the investment in established capacity depends on the co-state as well.
} 
the firm reduces capacities of the established product and hence $V^{m_{2}}$ increases $^{17}$.

Second, in the proof of Lemma 2 in Appendix A.2, we find that

$$
\frac{\partial V^{m_{2}}}{\partial K_{2}}\left(K_{1}^{F=0}, 0\right)=0
$$

which has an interesting economic intuition. In contrast to $m_{1}$, in $m_{2}$, the firm is able to invest in $K_{2}$. For $F=0$ at $K_{1}^{F=0}$ and elsewhere, there is no reason for waiting. But for higher $F>0$, waiting yields discounting of adoption costs while at $K_{1}^{F=0},(26)$ still holds and thus there is no gain from immediate switching to $m_{2}$ and investing in $K_{2}$. Thus, by postponing the product introduction, the incumbent can decrease the capacity of $K_{1}$ before switching such that $\frac{\partial V^{m_{2}}}{\partial K_{2}}\left(K_{1}^{l b}, 0\right)>0$, i.e. when switching, the marginal value of the new product's capacity is higher and hence there is an immediate gain from investment in $K_{2}$. Hence, the investment pattern in $m_{2}$ is affected, where due to the reduced capacity of the established product, the firm has stronger incentives to build-up capacities for the new product and the disinvestment in the established product is weaker ${ }^{18}$ than it would be without delay. Hence, in $m_{2}$, profits drop and are initially lower than in $m_{1}$ as there is a strong investment in capacities of the new product but sales increase only gradually for the new product. By delaying, the firm can postpone this drop in profits and enjoy 'high' profits in $m_{1}$. However, the drop in profits is stronger compared to immediate introduction.

\subsection{Bifurcation analysis}

We have a situation in mind where a new improved version of a product is launched which is a close substitute to the established product. This is reflected by a relatively high $\eta$ and low $\theta$. We do robustness checks with respect to those parameters in Section 4.3.1. The other parameter choices are very standard.

From Figure 3, it is clear that the value function is not concave in $K_{1}$ and hence does not satisfy the Arrow-Mangasarian sufficiency conditions. Thus, as mentioned

\footnotetext{
${ }^{17}$ This holds as long as the switching capacity $K_{1}^{l b}$ is greater than the maximal argument of $V^{m_{2}}$ which is true for the considered parameter setting.

${ }^{18}$ This is due to the increased marginal value of the established capacity.
} 
earlier, in this section we examine the qualitative properties of the steady states of the control problem with introduction option with respect to the parameter $F$. We start by drawing a bifurcation diagram of $m_{1}$ (Figure 5).

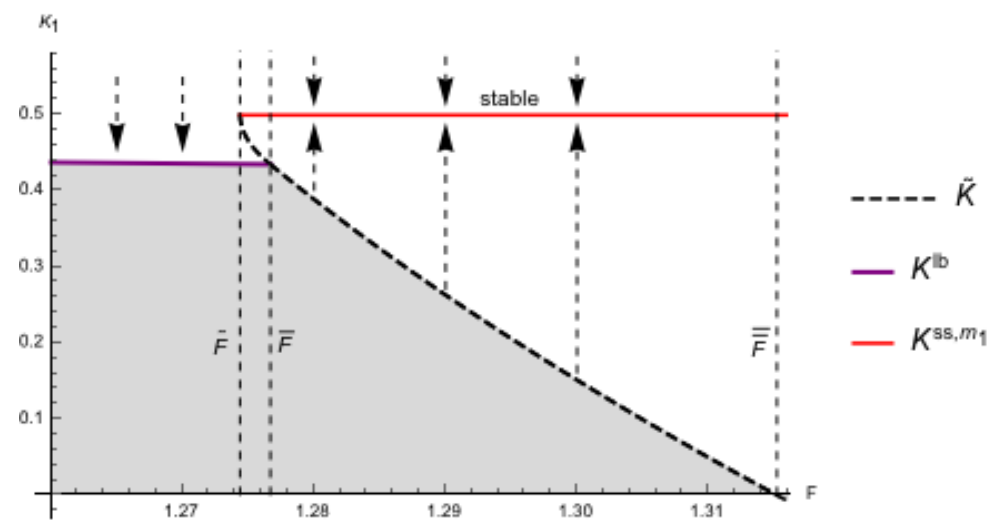

Figure 5: Bifurcation diagram of $m_{1}$.

The gray area is not present in $m_{1}$ since if the firm starts in that area or arrives there, it introduces the new product and hence is no more in $m_{1}$ but in $m_{2}$. As we are interested in characterizing dynamics in $m_{1}$ and in $m_{2}$ together, we draw a superimposed bifurcation diagram of both modes (cf. Hinloopen et al. (2017)) in Figure 6. For $F<\tilde{F}$, we have a unique stable steady state. No matter if

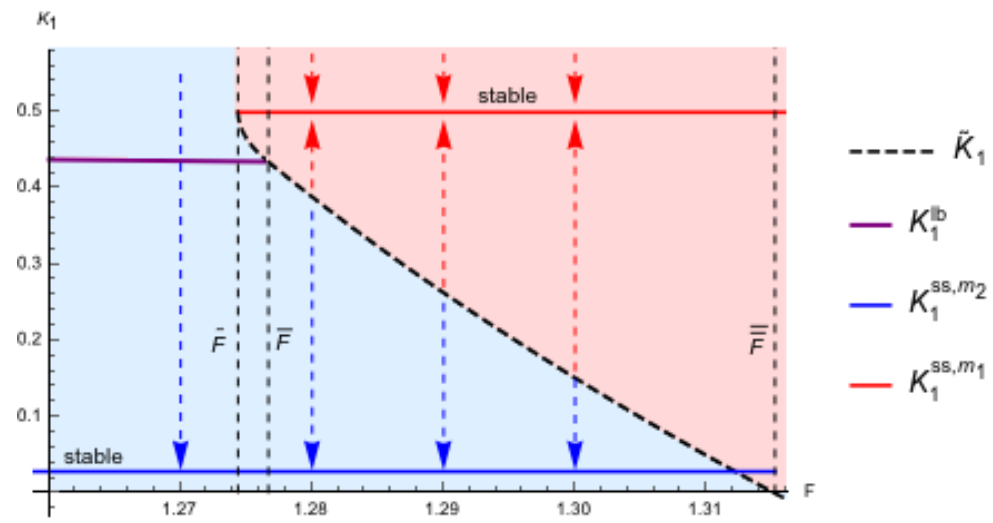

Figure 6: Superimposed diagram.

the firm delays product introduction or not, it will eventually arrive at the steady state level of $K_{1}$ in $m_{2}$ denoted by $K_{1}^{s s, m_{2}}$. As analyzed before, at $\tilde{F}$ there arises a second steady state where for initial capacities $\tilde{K}_{1} \leq K_{1} \leq K_{1}^{u b}$ (which are in the red area in Figure 6) the firm stays in $m_{1}$ and eventually arrives at $K_{1}^{s s, m_{1}}$. 
At $\overline{\bar{F}}$ the equilibrium point $K_{1}^{s s, m_{2}}$ vanishes and it remains only $K_{1}^{s s, m_{1}}$ for $F>\overline{\bar{F}}$ (see Figure 7).

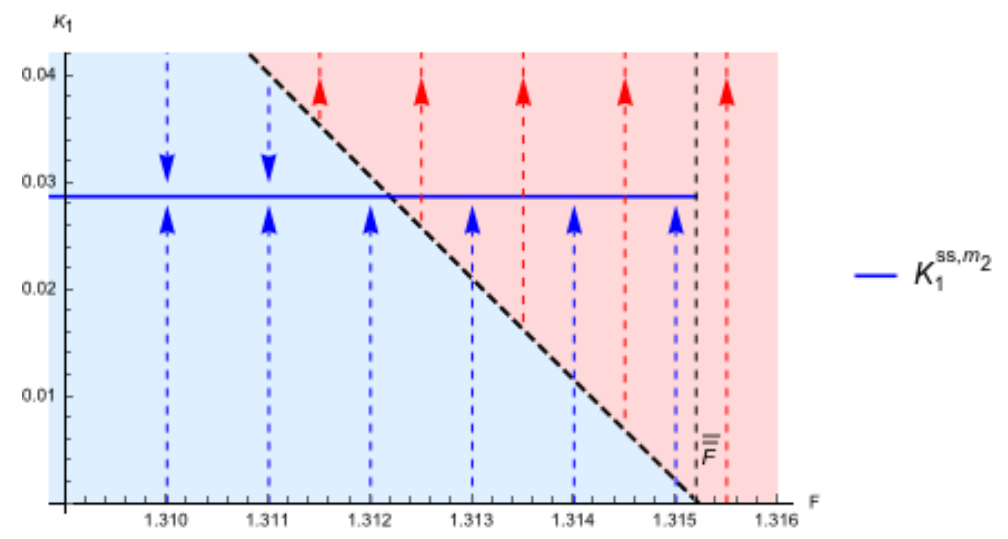

Figure 7: Dynamics around $\overline{\bar{F}}$.

Besides, we have a deformed pitchfork bifurcation which exhibits a hysteresis phenomenon where initially only one stable steady state exists and for higher $F$ a second equilibrium arises 'out of the blue sky', where a repelling curve separates the two basins of attraction (red and blue area) where for very high $F$ only the second equilibrium remains. The black dashed curve is the Skiba curve (which is repelling except at the two steady states where it is semi-stable). Note that for capacities on the Skiba curve in between the two steady states, optimal paths are moving in opposite directions but for capacities on the Skiba curve below $K_{1}^{s s, m_{2}}$ both optimal paths move in same direction (see Figure 7). Note that this is a superimposed diagram and not a bifurcation diagram in the classical sense and the latter is possible since there the firm either jumps immediately to $m_{2}$ or never, which means that we actually consider two disjoint optimal control problems where the mode can be interpreted as a further state variable.

\subsection{Characterization of optimal timing curves}

As discussed in Section 3, for $F \geq \tilde{F}, \tilde{K}_{1}$ separates finite and infinite solutions for the optimal introduction time. Thus, it jumps at $\tilde{K}_{1}$ to infinity. Hence, for $\tilde{K}_{1} \leq K_{1} \leq K_{1}^{u b}$, the value function of the problem with introduction option is equal to the value function of the problem without introduction option. 
We now investigate in detail what happens when $F$ approaches $\tilde{F}$. The graphs of the optimal introduction time are depicted in Figure 8. For low adoption costs,

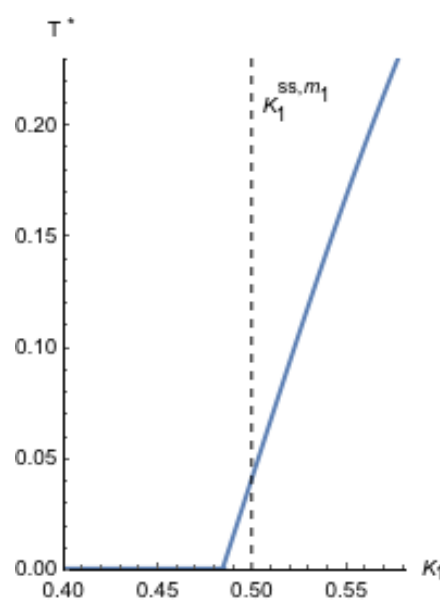

$F=1$

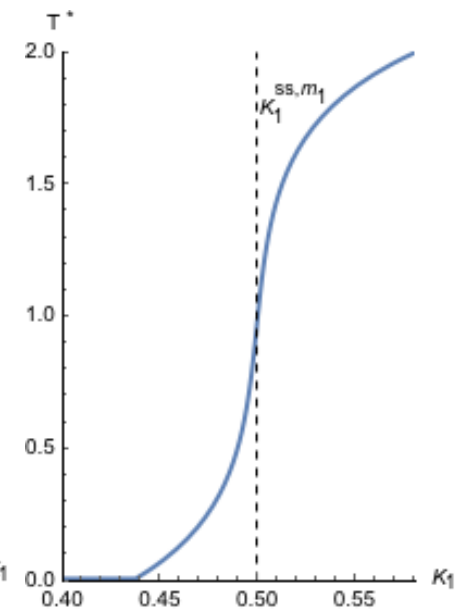

$F=1.274$

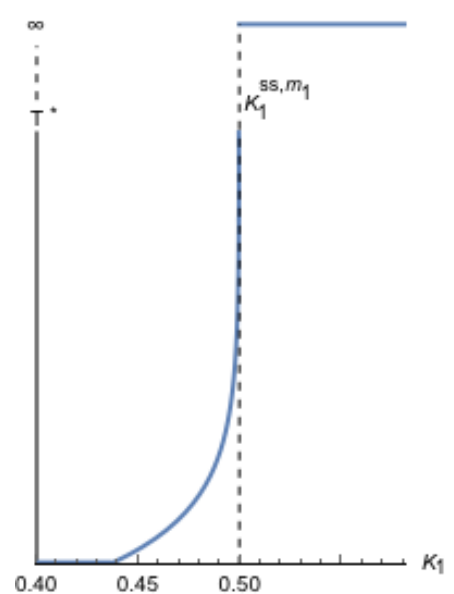

$F=\tilde{F} \approx 1.27437$

Figure 8: Optimal time of switching for increasing $F$.

the correspondence is concave for $K_{1} \geq K_{1}^{l b}$. As analyzed in Section 3, it is finite for low adoption costs whereas it becomes infinite at $\tilde{F}$ for $K_{1} \geq \tilde{K}_{1}=K_{1}^{s s, m 1}$. For $F$ approaching $\tilde{F}, T^{*}\left(K_{1}\right)$ becomes convex-concave and very steep at $K_{1}^{s s, m_{1}}$, i.e $K_{1}^{s s, m_{1}}$ becomes an inflection point (see Figure 8) which means that the firm decreases higher capacities and "stays around" $K_{1}^{s s, m_{1}}$ for a while until it starts decreasing again down to $K_{1}^{l b}$. Note that for $F<\tilde{F}, T^{*}\left(K_{1}\right)$ is finite everywhere, whereas at $\tilde{F}, T^{*}\left(K_{1}\right)$ is infinite for $K_{1} \geq K_{1}^{s s, m 1}$.

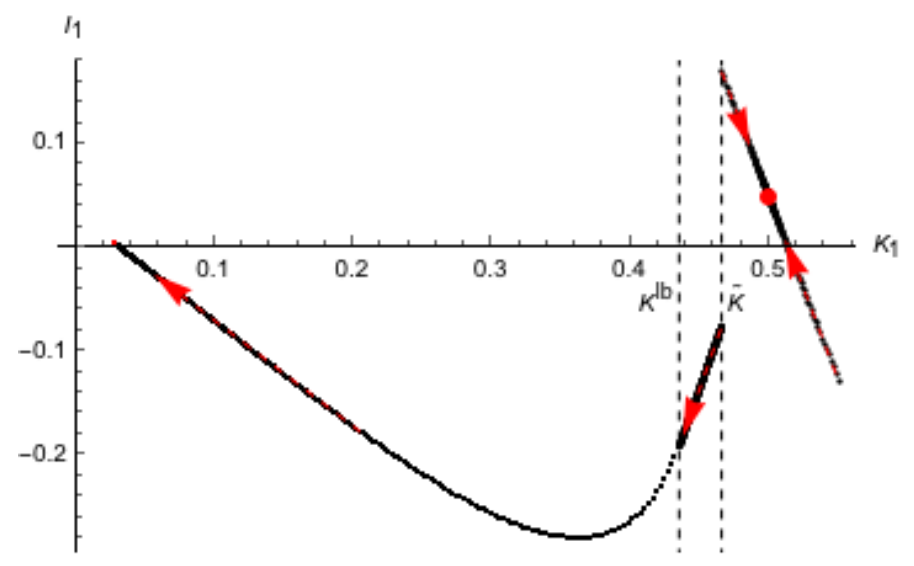

Figure 9: Capacity-investment dynamics for $F=1.275$.

Figure 9 depicts optimal curves in the $\left(K_{1}, I_{1}\right)$ space for the interesting case of 
intermediate adoption costs where $\tilde{K}_{1}$ separates the two basins of attraction. For $K_{1}^{l b}<K_{1}<\tilde{K}_{1}$, the firm decreases capacities down to $K^{l b}$ and introduces the new product. In $m_{2}$, it continues decreasing capacities of $K_{1}$ down to $K_{1}^{s s, m_{2}}$ while it builds up capacities for the new product up to $K_{2}^{s s, m_{2}}$.

\subsubsection{Effect of horizontal and vertical differentiation}

For decreasing degree of horizontal differentiation $\eta$, the products become more differentiated and thus the firm is expected to benefit from this. As both markets get more independent we expect that the firm is willing to introduce the new product earlier. Numerical experiments are in line with this intuition (see Figure 10). Analogously, for increasing $\theta$ we get similar results.
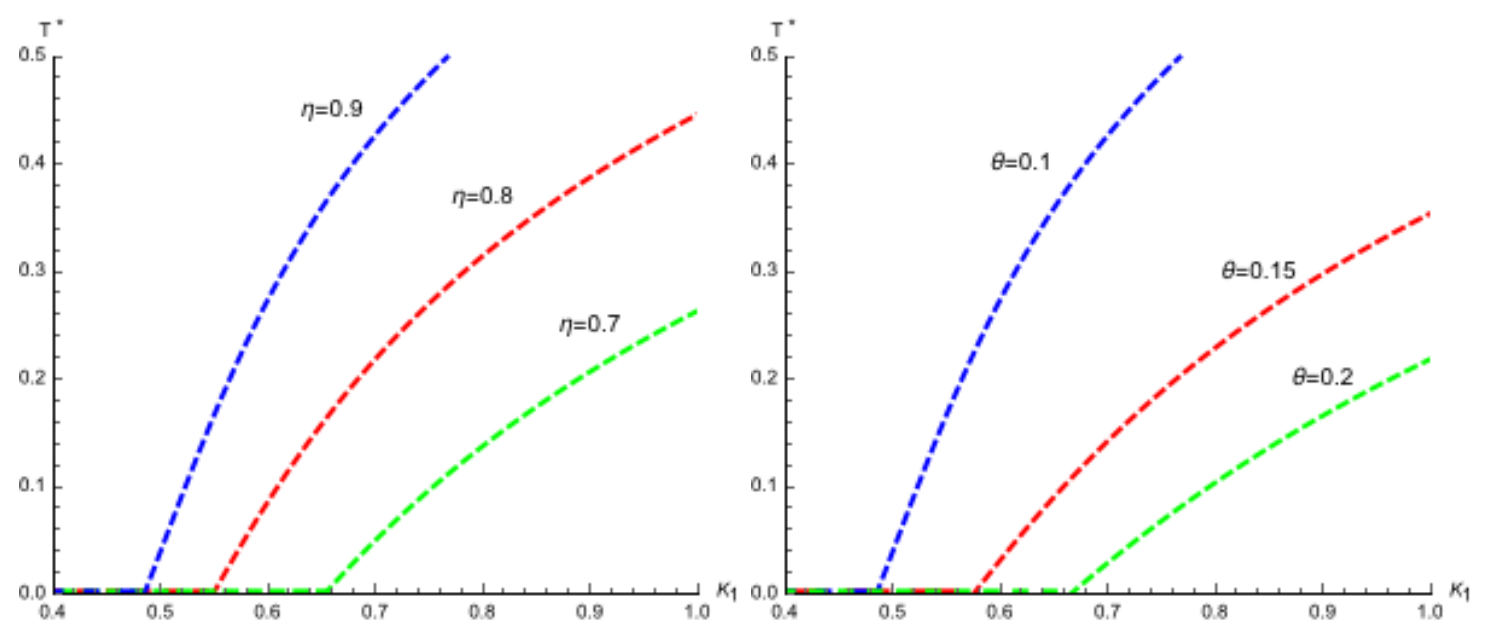

Figure 10: Optimal time of switching for different parameterizations of $\eta$ and $\theta$.

\section{Welfare implications}

For analyzing welfare implications, note that the inverse demand functions stem from the following utility function of the consumers where $M$ is the initial endowment:

$C S(t)=u\left(K_{1}, K_{2}\right)=K_{1}+(1+\theta) K_{2}-\frac{1}{2}\left(K_{1}^{2}+K_{2}^{2}\right)-\eta K_{1} K_{2}+\left(M-p_{1} K_{1}-p_{2} K_{2}\right)$. 
The welfare depends on the interpretation of adoption costs. If it is paid to the developer of the technology, then it is considered as a transfer and it is always profitable to introduce the new product immediately. But if it is considered as 'real' costs, then it has to be taken into account. In that case, the social planner maximizes the difference of consumer surplus and costs of investment and adoption: $\max _{T, I_{1}(t), I_{2}(t)} J=\int_{0}^{T} e^{-r t}\left(u\left(K_{1}, 0\right)-\frac{\gamma}{2} I_{1}^{2}\right) d t+\int_{T}^{\infty} e^{-r t}\left(u\left(K_{1}, K_{2}\right)-\frac{\gamma}{2}\left(I_{1}^{2}+I_{2}^{2}\right)\right) d t-e^{-r T} F$.

We expect that product introduction is favorable from a social point of view as in $m_{2}$, there is a new product of higher quality which affects the consumer only positively. For the given parameter setting, we find that delaying product introduction occurs only for very large $F$, in particular for $F>2.4492^{19}$. So, as expected, from the perspective of a social planner, it is optimal to introduce immediately for a wide range of $F$.

For the case of 'real' costs, the welfare difference of the situation of a profit maximizing firm and the situation where the firm is controlled by a social planner is depicted in Figure 11 for $K_{1}^{i n i}=K_{1}^{s s, m_{1}}$. The welfare loss is initially constant

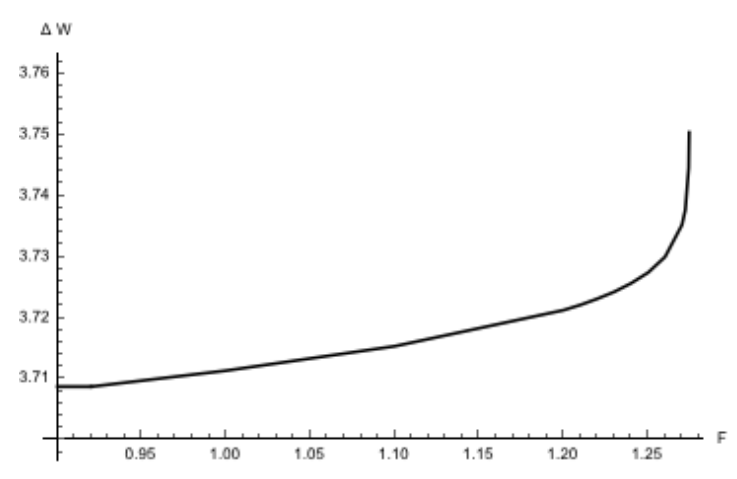

(a)

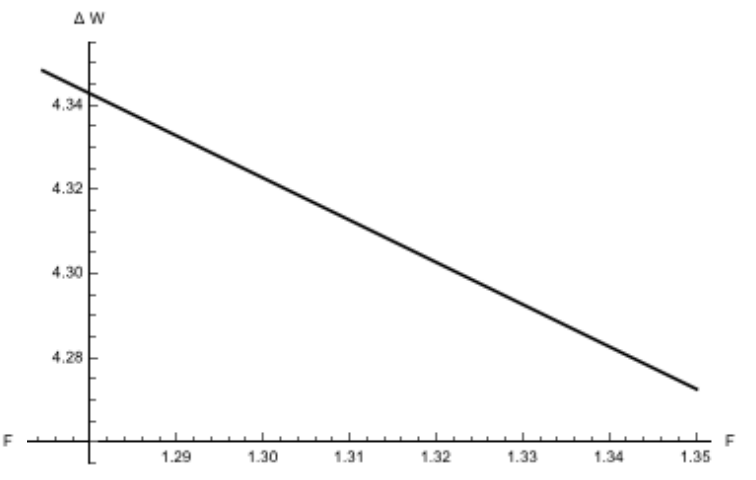

(b)

Figure 11: Welfare gain for $K_{1}=K_{1}^{s s, m_{1}}$. Parameters: $r=0.04, \delta=0.1, \eta=$ $0.9, \theta=0.1, \gamma=0.15$.

as in both situations, immediate introduction is optimal (as long as $K_{1}^{s s, m_{1}}<K_{1}^{l b}$ ) but at some critical $F$ (where $K_{1}^{l b}<K_{1}^{s s, m_{1}}$ ), the firm starts delaying the product

\footnotetext{
${ }^{19}$ Note that for the profit maximizing firm delay occurs even for $F=\epsilon, \epsilon>0$, which is substantially lower than 2.4492 .
} 
introduction which increases the welfare loss. However, for $F \geq \tilde{F}$, the welfare loss decreases (see Figure 11(b)) as the firm stays in $m_{1}$ where $F$ does not have an effect whereas the welfare for the social planner decreases as costs of switching to $m_{2}$ increases.

\section{Discussion of results and assumptions}

Somewhat surprising is that the first appearance of solutions where the firm stays with the established product is accompanied by a threshold point separating finite and infinite solutions for the terminal time. One might think that the rationale behind is that $m_{1}$ and $m_{2}$ are endogenously linked as in $m_{2}$ the number of products increases. But the phase-plane analysis (given in Appendix A.7) shows that this situation might occur even for a switch to an exogenously given mode, in particular whenever the terminal pair is on the unstable manifold.

From an economic perspective, delay was expected in order to discount adoption costs and increase the scrap value at the time of introduction. Our analysis shows that the decrease of established capacities is accompanied by a larger marginal value for the new product in $m_{2}$, i.e. investing in the capacities of the new product is stronger than it would be with immediate introduction.

In our analysis, we abstract from competition. However, a monopoly could turn into a competing environment if entry is possible. Thus, if there is a threat of possible entrants, we expect that this would accelerate product introductions.

Another issue is that we do not consider the phase of development of the new product. For the interpretation that the new product is developed by the incumbent himself, it is clear that the firm is not going to engage in R\&D activities if the product is not introduced eventually. In the case where the product is introduced with some delay, we expect that R\&D efforts would be less in the development phase which would have a similar impact on the introduction time.

For the interpretation of external developers generating a new technology where adoption costs mainly consist of buying the patent for the new technology, an alternative option to adoption costs which has to be paid once when the product 
is introduced, would be to consider fees per unit which has to be paid to the owner of the patent. There, as long as the fee per unit is constant and less than $\theta$, introduction would occur immediately since fees are paid continuously, so adoption costs are 'spread over time'.

We made the assumption that capacities are fully used, i.e. production equals sales. We believe that this assumption is of minor consequence to our results since in our model, there are no capacities for the new product in $T$ and investment in capacities has quadratic costs such that capacities are not build up as a 'lumpsum' but slowly while the capacity of the established product is reduced slowly. Moreover, in the case of delay, the incumbent starts reducing capacities even in $m_{1}$. A rigorous analysis of the full usage of capacity assumption yields that it is optimal to exploit full capacity if the following conditions hold:

$$
\begin{gathered}
2 K_{1}+\eta K_{2} \leq 1, \\
\eta K_{1}+2 K_{2} \leq 1+\theta .
\end{gathered}
$$

Numerical experiments suggest that conditions (29) and (30) seem to be satisfied for reasonable values of $K_{1}\left(\leq K_{1}^{s s, m_{1}}\right)^{20}$.

Furthermore, e.g. for decreasing demand, it is argued that in practice firms reduce prices in order to maintain production rather than reducing production due to contracts with employees and suppliers, even though such contracts are

\footnotetext{
${ }^{20}$ In the case of no horizontal and vertical differentiation, i.e. $\eta=1$ and $\theta=0$, conditions (29) and (30) are satisfied if

$$
K_{1} \geq \frac{1}{3} \wedge K_{2} \leq \frac{1}{3}
$$

or

$$
K_{1} \leq \frac{1}{3} \wedge K_{2} \geq \frac{1}{3}
$$

For our default parameter setting with $F=1.275,(31)$ and (32) are satisfied. In the case of horizontal and vertical differentiation, (29) and (30) are weakened. For higher $\theta$, the incumbent wants to build up capacities for the new product faster, but also to decrease capacities of the established product faster. For lower $\eta$, as products are more differentiated and competition of the established and the new product is weakened, investment in the new product's and disinvestment of the established product's capacities are slower. Thus, in both cases, we expect that (29) and (30) are not affected much.
} 
not modeled here (cf. Goyal and Netessine (2007)). However, counterexamples exist as well where firms have excess capacity e.g. for deterring entry (see Chicu (2012)).

This analysis focuses on the effect of adoption costs. However, for some products, not adoption costs but differences in production costs may be the main reason for firms to abstain from product introduction, in particular if the old and new product's production costs differ a lot. Apple had developed a mouse in 1979 whose production costs were too much such that Apple abstained from further development of this mouse and hence from introducing it (cf. Hinloopen et al. (2013)).

\section{Conclusion}

Using a fully dynamic framework we identify different scenarios where the firm's behavior depends crucially on the capacity of the established product and on the level of adoption costs. There is an interesting case where it is not optimal for the firm to introduce the new product immediately but to delay product introduction. By delay in time, adoption costs are discounted while the firm prepares for product introduction by reducing capacities on the established market which increases the marginal value of the established and new products' capacities. Moreover, the incumbent postpones investment in new capacity and hence benefits longer from high profits before product introduction. Noteworthy is the occurrence of Skiba points where the firm is indifferent in approaching different steady states which affects the number of products produced by the firm. We assumed that firms cannot invest in capacities beforehand. Allowing for investment before introduction might have an effect on the time of introduction, in particular we expect that this would accelerate product introduction while we think that qualitative results will be the same. Furthermore, we abstained from competition which would be the natural next step. 


\section{Acknowledgements}

The author would like to thank Herbert Dawid, Armin Schmutzler and participants at EARIE 2016 for many valuable comments and discussions. Support from the German Research Foundation (DFG) via CRC 1283 is gratefully acknowledged.

\section{A Appendix}

\section{A.1}

The canonical system is given by

$$
\begin{aligned}
\dot{K}_{1} & =\frac{\lambda}{\gamma}-\delta K_{1}, \\
\dot{\lambda} & =(r+\delta) \lambda-\left(1-2 K_{1}\right),
\end{aligned}
$$

and the isoclines are

$$
\begin{aligned}
\dot{K}_{1}=0 \quad \Leftrightarrow \quad \lambda=\delta \gamma K_{1}, \\
\dot{\lambda}=0 \quad \Leftrightarrow \quad \lambda=\frac{1-2 K_{1}}{r+\delta} .
\end{aligned}
$$

If the firm does not introduce the new product, i.e. for staying in $m_{1}$ infinitely, there is a unique steady state

$$
K_{1}^{s s, m_{1}}=\frac{1}{\delta \gamma(r+\delta)+2}, \quad \lambda^{s s, m_{1}}=\frac{\delta \gamma}{\delta \gamma(r+\delta)+2} .
$$

The steady state is a saddle point as the Jacobian is

$$
\left(\begin{array}{cc}
-\delta & \frac{1}{\gamma} \\
2 & r+\delta
\end{array}\right)
$$

with

$$
\operatorname{det} J=-\delta(r+\delta)-\frac{2}{\gamma}<0 .
$$

The eigenvalues are given by

$$
\mu_{1,2}=\frac{r}{2} \pm \sqrt{\left(\frac{r}{2}\right)^{2}+\delta(r+\delta)}
$$

so eigenvalues have different sign and the steady state is indeed a saddle point. 


\section{A.2}

Lemma 2. Condition (15) holds for

$$
\left(K_{1}^{*}\right)_{1,2}=-\frac{d}{f} \pm \sqrt{\frac{2 \gamma r F}{f^{2}}} .
$$

Proof.

Consider the terminal condition ${ }^{21}(15)$ :

$$
H\left(K_{1}^{*}, I_{1}^{*}, \lambda\left(T^{*}\right), T^{*}\right)=r S\left(K_{1}^{*}\right)
$$

$\Leftrightarrow$

$$
\left(1-K_{1}^{*}\right) K_{1}^{*}-\frac{\gamma}{2} I_{1}^{* 2}+\lambda\left(T^{*}\right)\left(I_{1}^{*}-\delta K_{1}^{*}\right)=r\left(V^{m_{2}}\left(K_{1}^{*}\right)-F\right)
$$

$\Leftrightarrow$

$$
\left(1-K_{1}^{*}\right) K_{1}^{*}-\frac{\gamma}{2} I_{1}^{* 2}+\frac{\partial V^{m_{2}}}{\partial K_{1}}\left(I_{1}^{*}-\delta K_{1}^{*}\right)=r\left(V^{m_{2}}\left(K_{1}^{*}\right)-F\right) .
$$

The HJB-equation in $m_{2}$ at $T^{*}$ is given by ${ }^{22}$

$$
\left(1-K_{1}^{*}\right) K_{1}^{*}-\frac{\gamma}{2}\left(I_{1}^{* 2}+I_{2}^{* 2}\right)+\frac{\partial V^{m_{2}}}{\partial K_{1}}\left(I_{1}^{*}-\delta K_{1}^{*}\right)+\frac{\partial V^{m_{2}}}{\partial K_{2}} I_{2}^{*}=r V^{m_{2}}\left(K_{1}^{*}\right) .
$$

For $I_{2}^{*}=\frac{V_{K_{2}}^{m_{2}}}{\gamma}$, we have:

$$
\left(1-K_{1}^{*}\right) K_{1}^{*}-\frac{\gamma}{2} I_{1}^{* 2}+\frac{\partial V^{m_{2}}}{\partial K_{1}}\left(I_{1}^{*}-\delta K_{1}^{*}\right)+\frac{1}{2 \gamma}\left(\frac{\partial V^{m_{2}}}{\partial K_{2}}\right)^{2}=r V^{m_{2}}\left(K_{1}^{*}\right) .
$$

Using (44) and (42) yields

$$
r F=\frac{1}{2 \gamma}\left(\frac{\partial V^{m_{2}}}{\partial K_{2}}\right)^{2}
$$

which under consideration of $K_{2}=0$ yields the two solutions

$$
K_{1}^{l b}:=-\frac{d}{f}-\sqrt{\frac{2 \gamma r F}{f^{2}}},
$$

and

$$
K_{1}^{u b}:=-\frac{d}{f}+\sqrt{\frac{2 \gamma r F}{f^{2}}} .
$$

\footnotetext{
${ }^{21}$ For convenience, we henceforth omit the dependence of state and control variables on $T^{*}$.

${ }^{22}$ Note that $F$ is paid for switching to $m_{2}$ and does not occur in $m_{2}$ anymore.
} 


\section{A.3}

Proof of Proposition 1. By Lemma 2 in Appendix A.2, we know that for $F=0$ the terminal condition of the Maximum Principle holds for $K_{1}^{F=0}$ and $H<r S$ for other values of capacity ${ }^{23}$. For $F>0, F$ occurs negatively on the right hand side of the terminal condition and only there. Thus, there arises an interval whose bounds are given by (46) and (47) wherein $H>r S$ (see Figure 12). For $K_{1}^{\text {ini }}$ outside the interval, the opposite holds. Hence, for $K_{1}^{i n i} \leq K_{1}^{l b}$, the interest on the salvage value is higher than the current value Hamiltonian. Thus, immediate introduction is optimal.

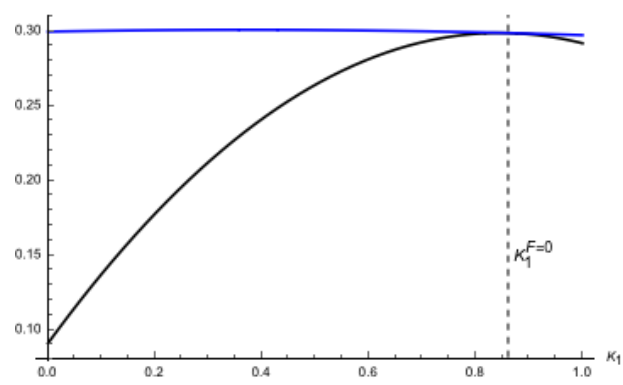

(a) $F=0$

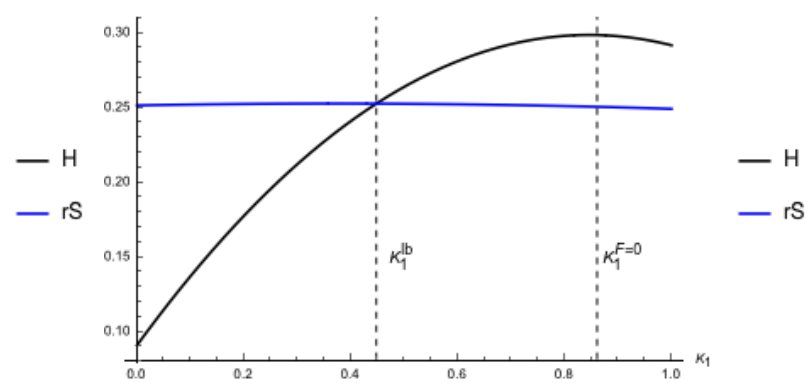

(b) $F=1.2$

Figure 12: Left hand side $(H)$ and right hand side $(r S)$ of terminal condition.

For $K_{1}^{l b}<K_{1} \leq K_{1}^{u b}$ the optimal switching capacity $K_{1}^{l b}$ has to be reached by the transversality condition. Thus the firm reduces capacities down to $K_{1}^{l b}$ and innovates.

\section{A.4}

Proof of Lemma 1. Taking the derivative of $K_{1}^{l b}$ with respect to $F$ yields

$$
\frac{\partial K^{l b}}{\partial F}=-\frac{2 \gamma r}{2 f^{2} \sqrt{\frac{2 \gamma r F}{f^{2}}}}=-\sqrt{\frac{\gamma r}{2 F f^{2}}}<0 .
$$

\footnotetext{
${ }^{23}$ Cf. Appendix A.2. For $F=0$, the square root in (39) vanishes and both solutions coincide. Moreover, note that for $F=0$, the only extra term in (44) in comparison to (42) is $\frac{1}{2 \gamma}\left(\frac{\partial V^{m_{2}}}{\partial K_{2}}\right)^{2}$ which is non-negative. Hence for all $K_{1}, H$ is less or equal than $r S$ (it is equal for $K_{1}^{l b}\left(=K_{1}^{u b}\right)$ as $\left.\frac{1}{2 \gamma}\left(\frac{\partial V^{m_{2}}}{\partial K_{2}}\right)^{2}=0\right)$.
} 


\section{A.5}

Lemma 3. $\exists ! \tilde{F}>0$ such that $\forall F \geq \tilde{F}, \exists K_{1}$ with $T^{*}\left(K_{1}\right)=\infty$, i.e. $V\left(K_{1}\right)=$ $V^{m_{1}}\left(K_{1}\right)$ and $\forall F<\tilde{F}, \nexists K_{1}$ with $T^{*}\left(K_{1}\right)=\infty$.

Proof. The value function of $m_{1}$ without the option to switch to $m_{2}$ is independent of $F$ whereas the value function of the control problem with introduction option is decreasing in $F$ due to the decreasing salvage value. Thus, there is some $\tilde{F}$ where the value function of the control problem with introduction option hits the value function of $m_{1}$ for the first time which is greater than 0 since for $F=0$, switching is costless and in $m_{2}$, there is the option of producing the new product which has a higher quality $(\theta>0)^{24}$.

\section{A.6}

Lemma 4. At $F=\tilde{F}$,

$$
K_{1}^{l b} \leq \tilde{K}_{1}
$$

holds.

Proof. Let $F=\tilde{F}$. Assume $\tilde{K}_{1}<K_{1}^{l b}$. Then, for $\tilde{K}_{1}, H<r S$, which yields that the unique solution is to switch to $m_{2}$ which contradicts $F=\tilde{F}$.

\section{A.7}

We first state the following lemma which is necessary for the proof of Proposition 2 .

Lemma 5. The dynamics at the terminal pair $\left(K_{1}^{l b}, \lambda(T)\right)$ are not $\dot{K}_{1}>0$ and $\dot{\lambda}>0$ simultaneously.

Proof. The terminal pair is determined by $H=r S$ and $\lambda(T)=S_{K_{1}}$. The line $\lambda(T)=S_{K_{1}}=b+c K_{1}$ has a positive ordinate $(b>0)$ as $K_{1}$ 's marginal value is

\footnotetext{
${ }^{24}$ Even for no vertical differentiation, introducing the new product is beneficial as the market is expanded and the firm is able to split the total quantity among the two products which yields a higher price (cf. Dawid et al. (2015)).
} 


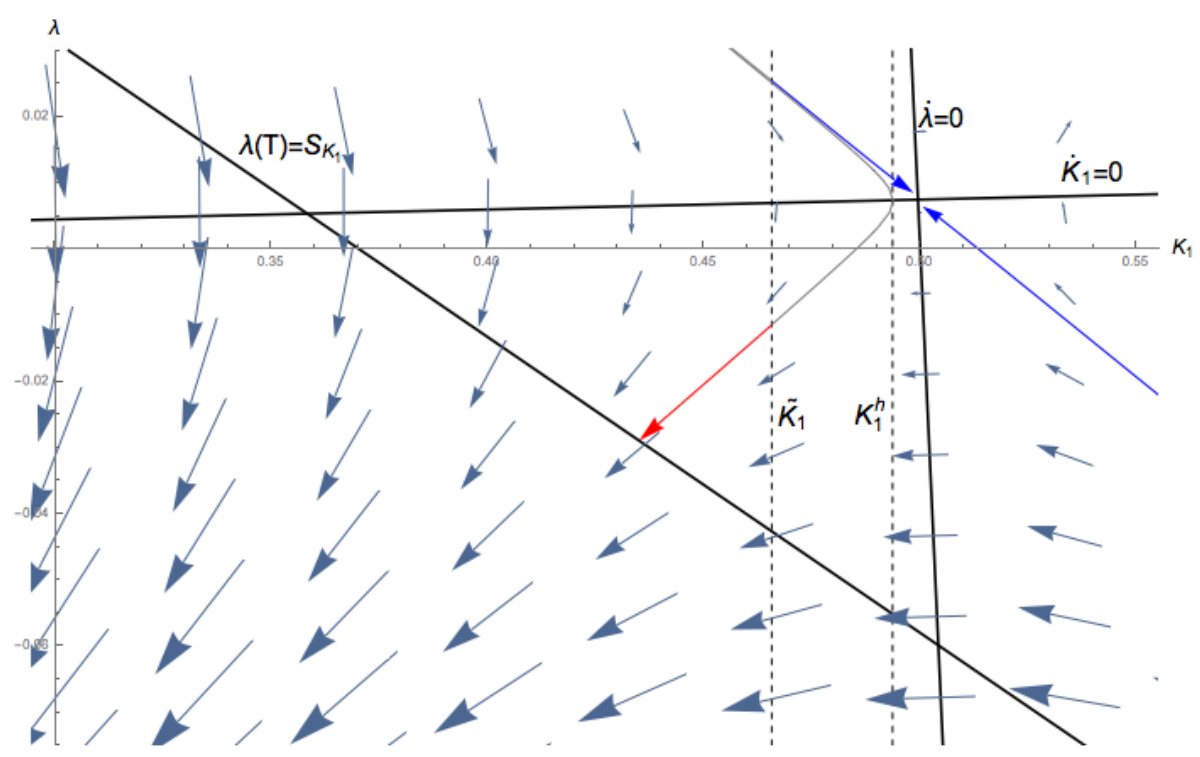

Figure 13: Vector plot for $F=1.275(>\tilde{F})$.

positive if there are no capacities installed. One might think that this line could pass through the area to the right-upper of the intersection point of $\dot{K}_{1}=0$ and $\dot{\lambda}=0$ where $\dot{K}_{1}>0$ and $\dot{\lambda}>0$ hold. This would yield different dynamics than studied so far. However, one can easily show that for terminal pairs in that area, there is no candidate for an optimal solution with $0<T^{*}<\infty$. In particular, for $K_{1}^{i n i}>K_{1}^{l b}$, there are either no candidate paths or only non-monotone paths arriving at the terminal pair which cannot be optimal ${ }^{25}$. Converging to the steady state of $m_{1}$ along the stable manifold is not optimal as well as time consistency is violated since for $K_{1}<K_{1}^{l b}, H<r S$ holds. Thus, there are no optimal paths for $K_{1}^{i n i}>K_{1}^{l b}$ which yields a contradiction and proves that this situation cannot occur. Moreover, the slope of the $\lambda(T)$ line is necessarily negative $(c<0)$, i.e. the marginal value of $K_{1}$ is decreasing as $\frac{\partial^{2} V^{m_{2}}}{\partial K_{1}^{2}}=c$.

Proof of Proposition 2. As the steady state of $m_{1}$ is a saddle-point, there is a stable and unstable manifold. If $T^{*}$ is finite but not zero, then the switching pair $\left(K_{1}(T), \lambda(T)\right)$ in the $\left(K_{1}, \lambda\right)$ space is derived from the condition $H=r S$ and the

\footnotetext{
${ }^{25}$ Non-monotone paths imply a set of Skiba points which generates fluctuating paths for $T^{*}=\infty$, which contradicts to the uniqueness property of the steady state of the infinite horizon problem.
} 
transversality condition $\lambda(T)=S_{K_{1}}$. As $F$ increases and $K_{1}^{l b}$ decreases, there is an $F$, where $\left(K_{1}^{l b}, \lambda(T)\right)$ is on the unstable manifold with $\dot{K}_{1}<0$ and $\dot{\lambda}<0^{26}$. Denote that $F$ by $F^{u n s}$. For arriving at that pair, the initial pair has to be on the unstable manifold. Thus, for all $K_{1} \geq K_{1}^{s s, m_{1}}$, there is no optimal path which leads to $\left(K_{1}^{l b}, \lambda(T)\right)$, i.e. for all $K_{1} \geq K_{1}^{s s, m_{1}}, T^{*}\left(K_{1}\right)=\infty$.

Next, we prove that $\tilde{F}=F^{u n s}$. Obviously, $\tilde{F} \leq F^{\text {uns }}{ }^{27}$. Assume $\tilde{F}<F^{\text {uns }}$. Then, by Lemma 1 , at $\tilde{F}$, the terminal pair is right to the unstable manifold. Denote for all possible terminal values $K_{1}(T)$ the value of the path which leads to the terminal pair by $V^{\text {term }}\left(K_{1}(t), K_{1}(T), F\right)$ which in this case exists for all $K_{1} \geq K_{1}(T)$ and for all $F<F^{u n s}$ and is continuous in $F$.

In order to avoid confusion, for an $F$, we denote the corresponding $K_{1}^{l b}$ by $K_{1}^{l b}(F)$. For $K_{1}^{i n i}>\tilde{K}_{1}$,

$$
V^{t e r m}\left(K_{1}^{i n i}, K_{1}^{l b}(\tilde{F}), \tilde{F}\right)<V^{m_{1}}\left(K_{1}^{i n i}\right)
$$

holds $^{28}$. Hence, $\exists F^{l}<\tilde{F}$ with

$$
V^{\text {term }}\left(K_{1}^{i n i}, K_{1}^{l b}\left(F^{l}\right), F^{l}\right)=V^{m_{1}}\left(K_{1}^{i n i}\right)
$$

which contradicts the minimality of $\tilde{F}$. Hence, the assumption $\tilde{F}<F^{u n s}$ was wrong and $\tilde{F}=F^{u n s}$ holds.

Now, we prove that $\tilde{K}_{1}$ is not less than $K_{1}^{s s, m_{1}}$ again by contradiction. Assume that $\tilde{K}_{1}<K_{1}^{s s, m_{1}}$. Then, consider $K_{1}^{\text {int }}$ for which $\tilde{K}_{1}<K_{1}^{\text {int }}<K_{1}^{s s, m_{1}}$ holds. For

\footnotetext{
${ }^{26}$ As shown in Lemma 5 , the dynamics at the terminal pair are not $\dot{K}_{1}>0$ and $\dot{\lambda}>0$ simultaneously. Hence, the line passes through the area where $\dot{K}_{1}<0$ and $\dot{\lambda}<0$ holds as it has a positive ordinate and negative slope.

${ }^{27}$ Note that for $F^{u n s}$ infinite solutions for $T$ exist. As $\tilde{F}$ is the minimal value of adoption costs for which infinite solutions exist, $\tilde{F} \leq F^{u n s}$ holds.

${ }^{28}$ It can not be $V^{\text {term }}\left(K_{1}^{i n i}, K_{1}^{l b}(F), F\right)=V^{m_{1}}\left(K_{1}^{i n i}\right)$ since for $K_{1}^{i n i} \geq \tilde{K}_{1}$, trajectories of the finite and infinite solution move in the same direction (as due to Lemma $4, K_{1}^{l b} \leq \tilde{K}_{1}$ ) and according to Proposition 1 in Caulkins et al. (2015), in that case, the trajectories have to coincide for all $t \in\left[0, T^{*}\left(K_{1}^{i n i}\right)\right]$ which is apparently not true. Moreover, $V^{t e r m}\left(K_{1}^{i n i}, K_{1}^{l b}(F), F\right)>V^{m_{1}}\left(K_{1}^{i n i}\right)$ cannot hold either since this leads to another solution for the problem without introduction option via moving to $\tilde{K}_{1}$ along the path corresponding to the finite solution of $T$ and switching at $\tilde{K}_{1}$ to the solution of the problem without introduction option.
} 
$F=\tilde{F}$, we have 29

$$
V^{t e r m}\left(K_{1}^{i n t}, K_{1}^{l b}(\tilde{F}), \tilde{F}\right)<V^{m_{1}}\left(K_{1}^{i n t}\right)
$$

Again, by continuity of $V^{\text {term }}$ in $F$, there exists an $F^{l}<\tilde{F}$ with

$$
V^{\text {term }}\left(K_{1}^{\text {int }}, K_{1}^{l b}\left(F^{l}\right), F^{l}\right)=V^{m_{1}}\left(K_{1}^{\text {int }}\right)
$$

which contradicts the minimality of $\tilde{F}$. Thus, $\tilde{K}_{1}=K_{1}^{s s, m_{1}}$ and it is a threshold point $^{30}$ where the firm is not indifferent.

\section{A.8}

Proof of Proposition 3. As $K_{1}^{l b}$ decreases with $F$, for $\tilde{F}<F<\bar{F}$, the terminal pair $\left(K_{1}(T), \lambda(T)\right)=\left(K_{1}^{l b}, \lambda(T)\right)$ is left to the unstable manifold (cf. proof of Proposition 2 in A.7). There, the dynamics are given by $\dot{K}_{1}<0$ and $\dot{\lambda}<0$. Starting at the terminal pair $\left(K_{1}^{l b}, \lambda(T)\right)$ and moving backwards along the arc leading to it, i.e. considering $V^{\text {term }}$ introduced in A.7 (cf. Figure 13), we can identify candidates for the optimal starting point for different $K_{1}^{i n i}$ 's. This arc hits the $\dot{K}_{1}=0$ line at some $K_{1}^{h}$. This is the highest $K_{1}$ for which a finite candidate $T$ exists since following the arc further gives further candidates for $K_{1}^{l b} \leq K_{1}<K_{1}^{h}$ as there is $\dot{K}_{1}>0$, which implies non-monotone paths for $K_{1}$ which can not be optimal (cf. Appendix A.7). Hence, $V^{t e r m}$ is well defined. For any $K_{1}<K_{1}^{s s, m_{1}}$, it is also possible to converge to the steady state of $m_{1}$ by following the stable arc of the steady state. Comparing values of both candidates by taking the upper curve of the value functions corresponding to both options we obtain the value function and the optimal strategies of the control problem with introduction option. Hence,

\footnotetext{
${ }^{29}$ Note that in this case, $V^{\text {term }}$ exists for $K_{1}<\tilde{K}_{1}$. Moreover, as this problem is time invariant and trajectories of the finite and infinite solution move in opposite directions and due to the monotonicity of the trajectory of the infinite solution (see Hartl (1987)), the trajectory of the finite solution is monotone as well and there can not be an overlap region, i.e. there is no interval of Skiba points (cf. Caulkins et al. (2015)). Thus, at $\tilde{F}$ for $K_{1}^{i n t}$, the infinite solution is the unique optimal solution.

${ }^{30}$ Here, a threshold point is characterized by having finite and infinite solutions for $T$ in every neighborhood (cf. Caulkins et al. (2015)).
} 
there is an indifference point $0<\tilde{K}_{1} \leq K_{1}^{h}$ where the firm is indifferent moving to the steady state along the stable manifold and moving to $K_{1}^{l b}$. Thus, $\tilde{K}_{1}$ is a Skiba point. As $F$ increases, $K_{1}^{l b}$ and $K_{1}^{h}$ decreases. Next, we prove that $\tilde{K}_{1}$ decreases as well by contradiction. For $F^{a}, F^{b} \in(\tilde{F}, \bar{F})$, with $F^{a}<F^{b}$, denote the corresponding indifference points by $\tilde{K}_{1}{ }^{a}$ and $\tilde{K}_{1}{ }^{b}$ and assume that $\tilde{K}_{1}{ }^{a} \leq \tilde{K}_{1}{ }^{b}$, i.e. $\tilde{K}_{1}$ is nondecreasing in $F$. Then,

$$
V^{m_{1}}\left(\tilde{K}_{1}^{b}\right)=V^{t e r m}\left(\tilde{K}_{1}^{b}, K_{1}^{l b}\left(F^{b}\right), F^{b}\right)<V^{t e r m}\left(\tilde{K}_{1}^{b}, K_{1}^{l b}\left(F^{a}\right), F^{a}\right) \leq V^{m_{1}}\left(\tilde{K}_{1}^{b}\right)
$$

which yields a contradiction ${ }^{31}$. Hence, $\tilde{K}_{1}$ is decreasing in $F$.

\section{References}

Caulkins, J. P., Feichtinger, G., Grass, D., Hartl, R. F., Kort, P. M., and Seidl, A. (2015). Skiba points in free end-time problems. Journal of Economic Dynamics and Control, 51:404-419.

Chandy, R. K. and Tellis, G. J. (2000). The Incumbent's Curse? Incumbency, Size, and Radical Product Innovation. Journal of Marketing, 64(3):1-17.

Chicu, M. (2012). Dynamic Investment and Deterrence in the U.S. Cement Industry. Job Market Paper.

Dawid, H., Keoula, M. Y., Kopel, M., and Kort, P. M. (2015). Product innovation incentives by an incumbent firm: A dynamic analysis. Journal of Economic Behavior \& Organization, 117:411-438.

Dixit, A. K. and Pindyck, R. S. (1994). Investment under uncertainty. Princeton: Princeton University Press.

Doraszelski, U. (2004). Innovations, improvements, and the optimal adoption of new technologies. Journal of Economic Dynamics and Control, 28(7):1461-1480.

\footnotetext{
${ }^{31}$ The last inequality is due to the following: $\tilde{K}_{1}{ }^{a} \leq \tilde{K}_{1}{ }^{b}$ and for $K_{1} \geq \tilde{K}_{1}{ }^{a}$, infinite solutions are optimal.
} 
Farzin, Y. H., Huisman, K. J. M., and Kort, P. M. (1998). Optimal timing of technology adoption. Journal of Economic Dynamics and Control, 22(5):779799.

Fudenberg, D. and Tirole, J. (1985). Preemptive and Rent Equalization in the Adoption of New Technology. The Review of Economics Studies, 52(3):383401.

Goyal, M. and Netessine, S. (2007). Strategic Technology Choice and Capacity Investment Under Demand Uncertainty. Management Science, 53(2):192-207.

Grass, D., Caulkins, J., Feichtinger, G., Tragler, G., and Behrens, D. (2008). Optimal control of nonlinear processes: With applications in drugs, corruption, and terror. Springer.

Hartl, R. F. (1987). A Simple Proof of the Monotonicity of the State Trajectories in Autonomous Control Problems *. Journal of Economic Theory, 215:211-215.

Hinloopen, J., Smrkolj, G., and Wagener, F. (2013). From mind to market: A global, dynamic analysis of R\&D. Journal of Economic Dynamics and Control, 37(12):2729-2754.

Hinloopen, J., Smrkolj, G., and Wagener, F. (2017). Research and Development Cooperatives and Market Collusion: A Global Dynamic Approach. Journal of Optimization Theory and Applications, 174(2):567-612.

Huisman, K. J. M. and Kort, P. M. (2015). Strategic capacity investment under uncertainty. RAND Journal of Economics, 46(2):376-408.

Kamien, M. I. and Schwartz, N. L. (1972). Some Economic Consequences of Anticipating Technical Advance. Western Economic Journal, 10(2):123-138.

Kreps, D. M. and Scheinkman, J. a. (1983). Quantity Precommitment and Bertrand Competition Yield Cournot Outcomes. The Bell Journal of Economics, $14(2): 326-337$. 
Long, N. V., Prieur, F., Tidball, M., and Puzon, K. (2017). Piecewise closedloop equilibria in differential games with regime switching strategies. Journal of Economic Dynamics and Control, 76:264-284.

Reinganum, J. F. (1981). On the Diffusion of New Technology: A Game Theoretic Approach. Review of Economic Studies, 48(3):395-406.

Skiba, A. K. (1978). Optimal Growth With a Convex-Concave Production Function. Econometrica, 46(3):527-539.

Wang, Q. H. and Hui, K. L. (2012). Delayed product introduction. In Decision Support Systems, volume 53, pages 870-880. 